\section{Journal of Epilepsy and \\ Clinical \\ Neurophysiology}

J Epilepsy Clin Neurophysiol 2011;17(3):100-105

\title{
Revisão Terminológica e Conceitual para Organização de Crises e Epilepsias: Relato da Comissão da ILAE de Classificação e Terminologia, 2005-2009. Novos Paradigmas?
}

\author{
Laura M. F. F. Guilhoto \\ Liga Internacional Contra Epilepsia
}

\begin{abstract}
RESUMO
Introdução: A organização das crises epilépticas e epilepsias vem sendo descrita em classificações organizadas pela Liga Internacional contra a Epilepsia (International League Against Epilepsy, ILAE) há cerca de meio século. Segundo estes documentos iniciais aperfeiçoados na década de 1980, as crises foram divididas em focais ou generalizadas, de acordo com o seu modo de início, ou em uma região específica do cérebro ou de forma bilateral, respectivamente. A etiologia das epilepsias foi considerada idiopática, sintomática ou criptogênica. Recentemente ampla discussão na comunidade científica surgiu após a publicação em 2010 de uma revisão terminológica e conceitual da Comissão de Classificação e Terminologia da ILAE. Objetivo/Método: Divulgação em Português do resumo e comentários da discussão da "Revisão terminológica e conceitual para organização de crises e epilepsias: Relato da Comissão de Classificação e Terminologia da ILAE, 2005-2009" a fim de apresentá-la aos profissionais da área de saúde dos países de língua portuguesa. Resultados: Os termos generalizado e focal foram redefinidos; crises que ocorrem em redes neuronais bilateralmente distribuídas que rapidamente as engajam são generalizadas; e aquelas que ocorrem dentro de redes delimitadas a um hemisfério ou discretamente localizadas ou mais amplamente distribuídas são focais. A classificação de crises generalizadas foi simplificada. As crises focais devem ser descritas de acordo com suas manifestações (por ex., discognitiva, motora, etc). Os conceitos generalizado e focal não se aplicam a síndromes eletroclínicas. Genético, metabólico-estrutural e desconhecido representam conceitos modificados para substituir os termos idiopático, simtomático e criptogênico, respectivamente. Nem todas as epilepsias são reconhecidas como síndromes eletroclínicas. A organização da epilepsia é feita pela especificadade, a saber, síndromes eletroclínicas, epilepsias não sindrômicas com causa estrutural ou metabólica, e epilepsias de causa desconhecida. Classes naturais (por ex. causa específica subjacente, idade de início, tipos associados de crises) ou agrupamentos pragmáticos (por ex. encefalopatia epiléptica, síndromes eletroclínicas auto-limitadas) podem servir tanto para organizar o conhecimento sobre formas reconhecidas de epilepsia como facilitar a identificação de outras novas. Várias opiniões dos especialistas internacionais sobre essa terminologia foram divulgadas e uma revisão ampliada será apresentada em 2013 pela Comissão de Classificação e Terminologia da ILAE. Conclusão: A Comissão de Classificação e Terminologia da ILAE (20052009) revisou conceitos, terminologia e abordagens para classificar crises e formas de epilepsia tendo publicado em 2010 os resultados do grupo de discussão vigentes nessa data e os resultados finais serão divulgados em 2013.
\end{abstract}

Palavras chave: epilepsia; classificação; síndrome; crise; organização.

\section{ABSTRACT}

Revised terminology and concepts for organization of seizures and epilepsies: Report of the ILAE Commission on Classification and Terminology, 2005-2009. New paradigms?

Introduction: The categorization of epileptic seizures and epilepsies has been described in organized Classifications by the International League Against Epilepsy (ILAE) in the last 50 years. According to these initial documents

\footnotetext{
${ }^{a}$ Membro da Comissão de Classificação das Crises e Epilepsias da Liga Internacional Contra Epilepsia (International League Against Epilepsy, ILAE) 2009-2013.
}

Received July 25, 2011; accepted Aug. 19, 2011. 
which have been reviewed in the $1980^{\text {ths }}$, epileptic seizures were divided in partial (focal) and generalized, regarding its onset type, either in one specific area in the brain or in a bilateral pattern, respectively. The etiology of the epilepsies were then considered to be idiopathic, symptomatic or criptogenic. Recently a broad discussion has emerged since the publication of the document of the Commission on Classification and Terminology of ILAE in 2010. Objetive/Method: Divulgation in Portuguese of the summary and comments of the "Revised terminology and concepts for organization of seizures and epilepsies: Report of the ILAE Commission on Classification and Terminology, 2005-2009" in order to present it to the Health personnel of the Portuguese Speaking countries. Results: Generalized and focal are redefined for seizures as occurring in and rapidly engaging bilaterally distributed networks (generalized) and within networks limited to one hemisphere and either discretely localized or more widely distributed (focal). Classification of generalized seizures is simplified. No natural classification for focal seizures exists; focal seizures should be described according to their manifestations (e.g., dyscognitive, motor, etc). The concepts of generalized and focal do not apply to electroclinical syndromes. Genetic, structural-metabolic, and unknown represent modified concepts to replace idiopathic, symptomatic, and cryptogenic, respectively. Not all epilepsies are recognized as electroclinical syndromes. Organization of forms of epilepsies is done by specificity, as follows: electroclinical syndromes, nonsyndromic epilepsies with structural-metabolic causes, and epilepsies of unknown cause. Natural classes (e.g., specific underlying cause, age at onset, associated seizure type), or pragmatic groupings (e.g., epileptic encephalopathies, self-limited electroclinical syndromes) may serve as the basis for organizing knowledge about recognized forms and facilitate identification of new ones. Several international specialist opinions about this report were divulged and a new report will be presented in 2013 by the Commission of Classification and Terminology of ILAE. Conclusion: The ILAE Commission on Classification and Terminology has revised concepts, terminology, and approaches for classifying seizures and forms of epilepsy and the results of the discussion group were published in 2010 and the final report will be released in 2013.

Key-words: epilepsy; classification; syndrome; seizure; organization.

\section{INTRODUÇÃO}

As classificações de crises epilépticas e epilepsias foram documentadas pela Liga Internacional contra a Epilepsia (International League Against Epilepsia, ILAE) a partir da década de 1960 culminando com as Classificações mais utilizadas até a presente data, as Classificações de 1981 e 1989, respectivamente. ${ }^{1-5}$

Segundo estes documentos iniciais aperfeiçoados na década de 1980 as crises foram divididas em focais ou generalizadas, de acordo com o seu modo de início, ou em uma região específica no cérebro ou de forma bilateral, respectivamente. A etiologia das epilepsias foi então considerada idiopática, sintomática ou criptogênica.

A partir de avanços tecnológicos ao longo dos anos, como o vídeo-EEG, que promoveu melhor conhecimento das manifestações ictais, outras propostas de classificação foram divulgadas pela ILAE ${ }^{6-8}$, e recentemente a proposta de revisão de terminologia de Berg et al. em nome da Comissão da ILAE de Terminologia e Classificação refletiu sobre as mudanças no conhecimento nas áreas de imagem e genética molecular no início do século $21 .{ }^{9}$ A partir desse documento vários aspectos nosológicos foram discutidos pela comunidade científica na área de epilepsia.

\section{OBJETIVO E MÉTODO}

Divulgação em Português do resumo e comentários da "Revisão terminológica e conceitual para organização de crises e epilepsias: Relato da Comissão de Classificação e Terminologia da Liga Internacional Contra a Epilepsia (International League Against Epilepsy, ILAE), 2005-2009" a fim de apresentá-la aos profissionais da área de saúde dos países de língua portuguesa. ${ }^{9}$

\section{RESULTADOS}

\section{Proposta de Berg et al., 2010}

A Comissão de Classificação e Terminologia da ILAE revisou conceitos, terminologia e abordagens para classificar crises e formas de epilepsia e após discussão publicou um documento em Março de 2010. ${ }^{9}$ Os termos generalizado e focal foram redefinidos; crises que ocorrem em redes neuronais bilateralmente distribuídas que rapidamente as engajam são generalizadas; e aquelas que ocorrem dentro de redes delimitadas a um hemisfério ou discretamente localizadas ou mais amplamente distribuídas são focais. A classificação de crises generalizadas foi simplificada (Tabela 1). Não há classificação natural para crises focais, que devem ser descritas de acordo com suas manifestações (por ex., discognitiva, focal motora), conforme descrito no glossário da ILAE de 2001 de Blume et al. ${ }^{10}$ (Tabela 2). Os conceitos generalizado e focal não se aplicam a síndromes eletroclínicas. Genético, metabólico-estrutural e desconhecido representam conceitos modificados para substituir os termos idiopático, simtomático e criptogênico, respectivamente. Nem todas as epilepsias são reconhecidas 
como síndromes eletroclínicas. A organização de formas de epilepsia é inicialmente feita pela especificidade: síndromes eletroclínicas, epilepsias não sindrômicas com causas estruturais ou metabólicas, e epilepsias de causa desconhecida (Tabela 3). Organização maior com estas divisões pode ser feita de uma maneira flexível dependendo do motivo. Classes naturais (por ex. causa específica subjacente, idade de início, tipos associados de crises) ou agrupamentos pragmáticos (por ex. encefalopatia epiléptica, síndromes autolimitadas eletroclínicas) podem servir como base para organizar o conhecimento sobre formas reconhecidas de epilepsia e facilitar a identificação de novas formas.

Tabela 1. Classificação das crises*.9

\begin{tabular}{l}
\hline Crises generalizadas \\
Tonico-clônica (em qualquer combinação) \\
Ausência \\
Típica \\
Atípica \\
Ausências com características especiais \\
Ausência Mioclônica \\
Mioclonia Palpebral \\
Mioclônica \\
Mioclônica \\
Mioclônica Atônica \\
Mioclônica Tônica \\
Clônica \\
Tônica \\
Atônica \\
Crises focais \\
Desconhecido \\
Espasmos epilépticos \\
* Crises que não podem ser claramente diagnosticadas nas categorias \\
precedentes não devem ser consideradas não classificadas até maiores \\
informações permitam o seu diagnóstico acurado. Entretanto esta não é uma \\
categoria de classificação.
\end{tabular}

Tabela 2. Descritores de crises focais de acordo com o grau de comprometimento durante a crise*. 9,10

\section{Sem comprometimento da consciência ou do contato \\ Com componentes observáveis motores ou autonômicos. Corresponde aproximadamente ao conceito anterior de crise parcial simples. \\ Pode ainda envolver apenas fenômenos subjetivos sensoriais ou psíquicos. \\ Corresponde ao conceito de aura, termo endossado no glossário de 2001. \\ Com comprometimento da consciência ou do contato \\ Corresponde ao conceito anterior de crise parcial complexa. \\ O temor "Discognitivo" é um termo que tem sido proposto para este conceito (Blume et al., 2001). \\ Evoluindo para uma crise convulsiva** bilateral (envolvendo componentes tônicos, clônicos, ou tônico-clônicos). Esta expressão substitui o termo "crise secundariamente generalizada".}

* Para mais descritores, veja Blume et al., 2001.

** O termo "convulsivo" foi considerado um termo leigo no glossário; entretanto, nota-se que ele é usado em várias formas na medicina e é traduzido de forma adequada em várias idiomas, sendo seu uso portanto endossado.
Tabela 3. Síndromes eletroclínicas e outras epilepsias. ${ }^{9}$

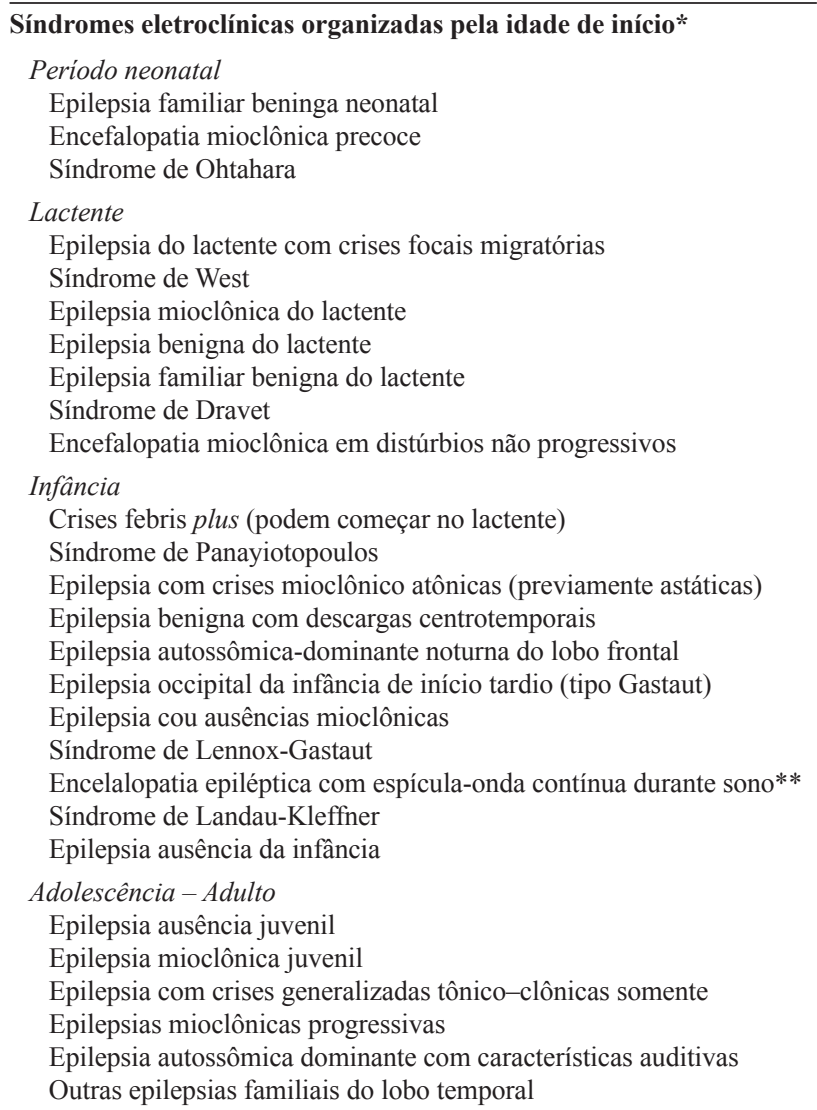

Relação menos específica com idade

Epilepsia familial focal com focos variáveis (infância à vida adulta) Epilepsias reflexas

\section{Constelações distintas}

Epilepsia mesial temporal com esclerose hipocampal

Síndrome de Rasmussen

Crises gelásticas com hamartoma hipotalâmico

Epilepsia-hemiconvulsão-hemiplegia

Epilepsias que não se enquadram em nenhuma destas categorais diagnósticas podem ser distinguidas inicialmente na presença ou ausência de condição estrutural ou metabólica (causa presumida) e no modo primário do início de crise (generalizado vs. focal)

\section{Epilepsias atribuídas a causa estrutural-metabólica}

Malformações do desenvolvimento cortical (hemimegalencefalia, heterotopia, etc.)

Síndromes neurocutâeas (complexo esclerose tuberosa, Sturge-Weber, etc.)

Tumor

Infecção

Trauma

Angioma

Insultos perinatais

Acidente vascular cerebral

Etc.

\section{Epilepsias de causa desconhecida}

Condições com crises epilépticas que são tradicionalmente não diagnosticadas como uma forma de epilepsia per si

Crises benignas neonatais Crises febris

* Este agrupamento de síndromes eletroclínicas não reflete etiologia ** Algumas vezes referido como estado de mal epiléptico elétrico durante sono lento. 


\section{Discussão da proposta de Berg et al., 2010}

Esta revisão terminológica recebeu vários comentários antes e após sua publicação. Shorvon propôs uma classificação da epilepsia baseada nos aspectos etiológicos, dividindo-a em 4 categorias, idiopática, sintomática, provocada e criptogênica, referindo problemas associados com a atribuição da causa, fatores de risco e da conceituação de epilepsia como doença e não como sintoma. ${ }^{11-2}$

Berg \& Scheffer em artigo sumarizado sobre o tema em 2011 recomendaram a utilização dos termos focal e generalizado para "redes neuronais" e o seu abandono para a classificação das epilepsias, devido à dificuldade da dicotomia existente entre focal e generalizado em algumas situações como nas encefalopatias epilépticas da infância. Neste artigo estas autoras salientaram também a inadequação dos termos idiopático, sintomático e criptogênico, por causa das mudanças ocorridas nas últimas décadas em relação aos métodos de imagem e genética molecular. Desta forma propõem para etiologia os termos, genético, estrutural/metabólico e desconhecida. Genético se aplica às epilepsias que são resultado direto de um defeito genético (gene ou mecanismo identificado) ou ainda a síndromes eletroclínicas nas quais estudos de gêmeos ou de segregação familiar mostram evidência reprodutível de base genética, como por exemplo, nas canalopatias. As formas de epilepsia com etiologia estrutural/metabólica são secundárias à condição estrutural ou metabólica, que eventualmente pode ter causa genética conhecida ou não. A etiologia desconhecida inclui os casos em que, até o momento, a causa não foi identificada pelos métodos diagnósticos. Berg \& Scheffer sugerem também nesse artigo o abandono da classificação rígida de 1989 e a utilização da classificação baseada em grupamentos como, por exemplo, por tipo de crises, padrão de EEG, alteração genética (Ex. mutações SLC2A1, SCN1A) ou ainda mecanismo envolvido (Ex. via mTOR). Salientam ainda que algumas síndromes eletroclínicas devem permanecer em evidência diagnóstica como, por exemplo, a Síndrome de West, cuja identificação é importante para manejo clínico, independente da classificação adotada. Segundo essas autoras o uso do glossário da ILAE de 2001 é um instrumento útil que deverá ser utilizado na classificação das crises focais, pois fornece maior descrição da fenomenologia clínica do que os termos anteriores "crise parcial simples e complexa". Termos também discutidos foram "benigno" e "catastrófico", que foram substituídos respectivamente por "auto-limitado" e "não farmacorresponsivo", sendo enfatizados os termos "encefalopatia epiléptica" que descrevem a deterioração neuropsíquica decorrente da atividade epiléptica, cujo diagnóstico e manejo se tornam prioritários. ${ }^{13-4}$

Vários posicionamentos surgiram dos presidentes anteriores da ILAE. Engel presidente da ILAE no período de 1997 a 2001 salientou a importância de se adicionar categoria de epilepsia considerada cirurgicamente tratável. ${ }^{15}$ Wolf, presidente de 2005 a 2009 divulga a idéia de epilepsias de sistemas, que envolvem redes neuronais responsáveis por funções fisiológicas no período interictal. ${ }^{16}$ Moshe, presidente da ILAE no período de 2009 a 2013 sugeriu manutenção das discussões até a divulgação de um novo documento de revisão no $30^{\circ}$ Congresso Internacional de Epilepsia em 2013. ${ }^{17}$ Jackson considerou que terminologias antigas fazem com que o conhecimento fique ancorado e retarde o progresso das descobertas e avanços científicos. ${ }^{18}$ Este autor considerou que as proposições de Berg et al. e Shorvon estão dirigidas no mesmo sentido e que no entanto novas terminologias são necessárias para indicar as mudanças ocorridas, não se devendo discutir, segundo este autor, apenas as palavras, mas os novos caminhos a serem tomados. ${ }^{18}$ Durante o $29^{\circ}$ Congresso Internacional de Epilepsia em Agosto de 2011 a Comissão de Classificação e Terminologia da ILAE divulgou um diagrama resumo da proposta de classificação para facilitar a futura discussão ${ }^{19}$ (Figura 1).

\section{CONCLUSÃO}

A Comissão de Classificação e Terminologia da ILAE (2005-2009) revisou conceitos, terminologia e abordagens para classificar crises e formas de epilepsia tendo publicado em 2010 os resultados do grupo de discussão vigentes até essa data e os resultados finais serão divulgados em 2013. 


\section{A- Classificação de Crises}

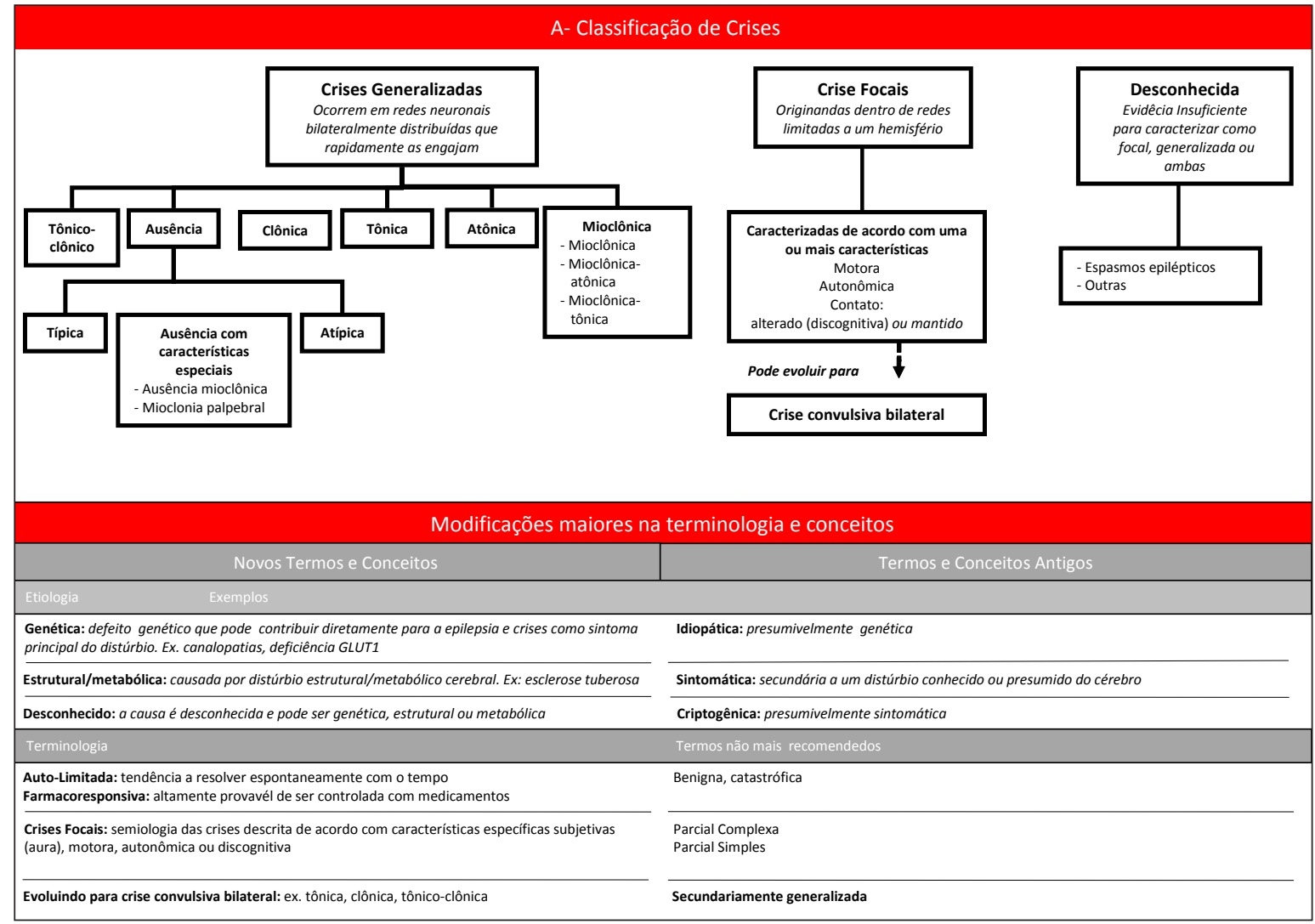

\section{B- Síndromes Eletroclínicas e outras Epilepsias}

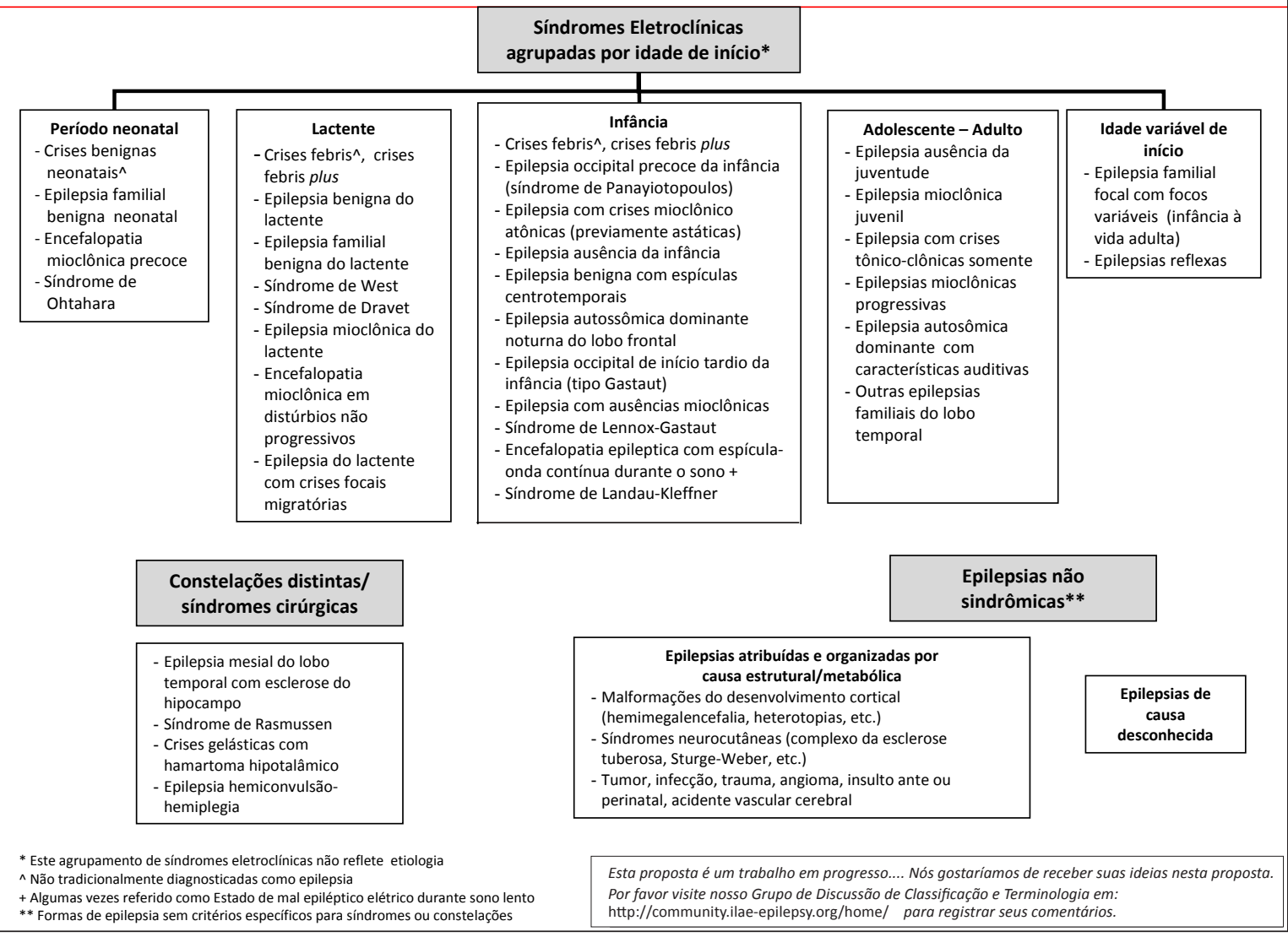

Figura 1 A e B. Diagrama resumo da Revisão terminológica para organização de crises e epilepsias da ILAE de 2010 divulgado durante o $29^{\circ}$ Congresso da ILAE/IBE em agosto de 2011.9-10,19-20 


\section{REFERÊNCIAS}

1. Gastaut H. Clinical and electroencephalographical classification of epileptic seizures. Epilepsia 1969;10:Suppl:2-13.

2. Gastaut H. Classification of the epilepsies. Proposal for an international classification. Epilepsia 1969;10:Suppl:14-21.

3. Proposal for revised clinical and electroencephalographic classification of epileptic seizures. From the Commission on Classification and Terminology of the International League Against Epilepsy. Epilepsia 1981;22:489-501.

4. Commission on Classification and Terminology of the International League Against Epilepsy. Proposal for classification of epilepsies and epileptic syndromes. Epilepsia 1985;26:268-78.

5. Proposal for revised classification of epilepsies and epileptic syndromes. Commission on Classification and Terminology of the International League Against Epilepsy. Epilepsia 1989;30:389-99.

6. Lüders H, Acharya J, Baumgartner C, Benbadis S, Bleasel A, Burgess R, Dinner DS, Ebner A, Foldvary N, Geller E, Hamer H, Holthausen H, Kotagal P, Morris H, Meencke HJ, Noachtar S, Rosenow F, Sakamoto A, Steinhoff BJ, Tuxhorn I, Wyllie E. A new epileptic seizure classification based exclusively on ictal semiology. Acta Neurol Scand. 1999;99:137-41. Review.

7. Engel J Jr. A proposed diagnostic scheme for people with epileptic seizures and with epilepsy: report of the ILAE Task Force on Classification and Terminology. Epilepsia 2001;42:796-803.

8. Engel J Jr. Report of the ILAE classification core group. Epilepsia 2006;47:1558-68.

9. Berg AT, Berkovic SF, Brodie MJ, Buchhalter J, Cross JH, van Emde Boas W, Engel J, French J, Glauser TA, Mathern GW, Moshe SL, Nordli D, Plouin P, Scheffer IE. Revised terminology and concepts for organization of seizures and epilepsies: Report of the ILAE Commission on Classification and Terminology, 2005-2009. Epilepsia 2010; 51:676-85.
10. Blume WT, Luders HO, Mizrahi E, Tassinari C, van Emde Boas W, Engel J. Glossary of descriptive terminology for ictal semiology: report of the ILAE task force on classification and terminology. Epilepsia 2001;42:1212-8.

11. Shorvon SD. The etiologic classification of epilepsy. Epilepsia 2011;52:1052-7.

12. Shorvon SD. Using etiology as one axis of classification. Epilepsia 2011;52:1208-9.

13. Berg AT, Scheffer IE. New concepts in classification of the epilepsies: Entering the 21st century. Epilepsia 2011; 52:1058-62.

14. Berg AT, Scheffer IE. What is at stake in a classification? Epilepsia 2011; 52:1205-8.

15. Engel J Jr. The etiologic classification of epilepsy. Epilepsia 2011;52:1195-7; discussion 1205-9.

16. Wolf P. Networks and systems, conceptualizations, and research. Epilepsia 2011;52:1198-200; discussion 1205-9.

17. Moshé SL. In support of the ILAE Commission classification proposal. Epilepsia 2011;52:1200-1; discussion 1205-9.

18. Jackson G. Classification of the epilepsies 2011. Epilepsia 2011; 52:1203-4; discussion 1205-9.

19. International League Against Epilepsy. Quick Overview of the 2010 proposal for organization of the epilepsies. 2011. Disponível em: <http://www.ilae-epilepsy.org/Visitors/Centre/ctf/documents/ ILAEHandoutV10_000.pdf $>$. Acesso em 01/09/2011.

20. Berg AT, Cross JH. Towards a modern classification of the epilepsies? Lancet 2010:9;459-61.

Endereço para correspondência:

Laura M. F. Ferreira Guilhoto

Associação Brasileira de Epilepsia

Rua Pedro de Toledo, 740 - Vila Clementino

CEP 04023-900, São Paulo, SP, Brasil

Fone: (11)5549-3819

E-mail: <lauragui@gmail.com> 